HNO 2021 $69: 615-622$

https://doi.org/10.1007/s00106-021-01015-2

Angenommen: 22. Januar 2021

Online publiziert: 23. Februar 2021

(c) Springer Medizin Verlag GmbH, ein Teil von Springer Nature 2021

\author{
M. Herzog $g^{1,2} \mathbb{D} \cdot$ A. G. Beule ${ }^{3,4} \cdot$ J.-C. Lüers ${ }^{5} \cdot$ O. Guntinas-Lichius ${ }^{6} \cdot$ D. Grafmans ${ }^{1} \cdot$ \\ T. Deitmer ${ }^{7}$ \\ ' Klinik für Hals-Nasen-Ohrenheilkunde, Kopf- und Halschirurgie, Carl-Thiem-Klinikum, Cottbus, \\ Deutschland \\ ${ }^{2}$ Klinik und Poliklinik für Hals-Nasen-Ohren-Heilkunde, Kopf- und Halschirurgie, Martin-Luther-Universität \\ Halle-Wittenberg, Halle (Saale), Deutschland \\ ${ }^{3}$ Klinik für Hals-, Nasen- und Ohrenheilkunde, Universitätsklinikum Münster, Münster, Deutschland \\ ${ }^{4}$ Klinik und Poliklinik für Hals-, Nasen-, Ohrenkrankheiten, Kopf- und Halschirurgie, Universitätsmedizin \\ Greifswald, Greifswald, Deutschland \\ ${ }^{5}$ Klinik und Poliklinik für Hals-, Nasen- und Ohrenheilkunde, Medizinische Fakultät, Uniklinik Köln, Köln, \\ Deutschland \\ ${ }^{6}$ Klinik und Poliklinik für HNO-Heilkunde, Universitätsklinikum Jena, Jena, Deutschland \\ ${ }^{7}$ Deutsche Gesellschaft für HNO-Heilkunde, Kopf- und Hals-Chirurgie e.V., Bonn, Deutschland
}

\title{
Das erste Jahr der Pandemie mit SARS-CoV-2 - Auswirkungen auf die Hals-Nasen-Ohren-Heilkunde
}

Kontext beleuchten und Gedankenanstöße für den zukünftigen Umgang mit dem Erreger aufzeigen.

\section{Methode}

Es erfolgte eine Recherche in den Datenbanken PubMed, medRxiv und bioRxiv (zuletzt am 28.11.2020) nach relevanter Literatur (deutsch, englisch) zu den Themen HNO-Heilkunde (ENT, ORL), Infektionsrisiko („risk of infection“), Corona, COVID-19, SARS-CoV-2 Aerosol, Gesundheitspersonal („health care professional") sowie im Deutschen Ärzteblatt und auf den Website des Robert KochInstituts und der Johns Hopkins University.

\section{Aerosolexposition und Infektionsrisiken bei Hals- Nasen-Ohren-ärztlichen Tätigkeiten}

Für den Bereich der Hals-Nasen-OhrenHeilkunde wird für die folgenden Tätigkeiten ein erhöhtes Risiko durch Aerosolexposition vermutet oder wurde bereits nachgewiesen.

1. Allgemeine Hals-Nasen-Ohren-
ärztliche Untersuchung
Für die Untersuchung der oberen Luftwege wird ein erhöhtes Infektionsrisiko mit SARS-CoV-2 beschrieben [14, 30, 35]. Insbesondere bei der starren oder flexiblen Endoskopie der Nase, des Nasenrachenraums, des Oro-/Hypopharynx und des Larynx wird von einer Aerosolbildung ausgegangen[18, 38].

2. Rhino-, Sinus- und Rhinobasischirurgie Durch die Manipulation an den Schleimhäuten, die als am stärksten virustragend identifiziert wurden, kommt es zu einer noch stärkeren Aerosolbildung in der näheren und auch weiteren Umgebung, sodass der komplette Operationsraum als kontaminiert angesehen werden sollte [10, 33, 48, 49]. Besonders die Verwendung von Bohrern oder Shavern („powered instrumentation“) soll die Aerosolbildung fördern [33]. Die parallele Absaugung (z. B. durch die kontralaterale Nase) wurde zur Verringerung der Aerosolbelastung empfohlen [24].

3. Otochirurgie Da das Mittelohr und das Mastoid mit dem Nasenrachen verbunden sind, stehen diese Strukturen im Jahr nach Ausbruch im internationalen 
Verdacht, mit SARS-CoV-2 besiedelt zu sein. In geringem Maß konnte eine Virusexpression in Mittelohren und Mastoiden bei 3 Obduktionen bei COVID-19-Patienten nachgewiesen werden [11]. Durch Bohrarbeiten könnte es zu einer Aerosolausbreitung kommen. [5, 7]. Es gilt hierbei jedoch zu beachten, dass die Datenlage zu diesem Aspekt dürftig ist.

4. Tracheostomie

Von vielen Autoren wird über die Gefahr der Aerosolfreisetzung im Rahmen einer Tracheostomie berichtet [28]. In Kliniken tätige HNOÄrzte waren bereits in der ersten Infektionswelle mit der Notwendigkeit konfrontiert, intubierte COVID-19Patienten zu tracheotomieren, und HNO-Fachgesellschaften entwarfen zügig Handlungsempfehlungen für ihre Mitglieder [8, 9, 12, 19, 27, 37].

\section{Schutzkonzepte gegen die Infektion mit dem Erreger SARS- CoV-2}

Insbesondere am Anfang der Pandemie im Frühjahr 2020 waren Empfehlungen $\mathrm{zu}$ bestimmten Sachverhalten verständlicherweise hauptsächlich auf Beobachtungen und Erfahrungen kleinerer Personenkreise zurückzuführen (z. B. [4, 45]). Deren Veröffentlichung auf Plattformen der Fachgesellschaften (z.B. HNO-CORONA-NEWS-TICKER der DGHNO-KHC) war dennoch hilfreich für unser klinisches Handeln zu Beginn der Pandemie.

Der Kernpunkt aller Empfehlungen bestand und besteht weiterhin in einem adäquaten Schutz für die Akteure im Gesundheitssystem und in der HalsNasen-Ohren-Heilkunde im Speziellen. Insbesondere die persönliche Schutzausrüstung, bestehend aus suffizienten Schutzmasken (FFP2/3), Schutzbrille, Kittel, Haube und Handschuhen, stand und steht hierbei im Mittelpunkt der Forderungen. Die Verwendung von Gebläsefiltergeräten (PAPR - „powered airpurifying respirator") wurde in diesem Zusammenhang bereits früh angeregt. Es fehlt allerdings bis heute Evidenz, dass diese Geräte auch zu einem erhöh- ten Schutz gegenüber SARS-CoV-2 im Vergleich zu FFP3-Masken beitragen. Es wird deswegen weder für klinische Routinemaßnahmen noch für Hoch-RisikoProzeduren empfohlen, PAPR anstatt FFP3-Masken zu verwenden [2, 26]. Auf der anderen Seite scheinen PAPR einen höheren Tragkomfort zu besitzen [25], und sie bieten sich bei stark Aerosol generierenden Eingriffen oder längerem Kontakt zu COVID-19-Patienten (z.B. Intensivverlegungen zwischen Kliniken) an. Nach persönlichen Informationen der Autoren wird dies in einigen Klinika in Deutschland praktiziert.

Parallel wurde die lokale Instillation von UV-Lampen und der regelmäßige Luftaustausch („Stoßlüften“) zur Minderung der Aerosollast empfohlen [32], wobei baulich bedingt in bestimmten Bereichen, wie einem Zentral-OP, die Umrüstung einer vorhandenen Klimaanlage eine komplexe Aufgabe darstellt.

Ein weiterer Ansatz, der zu einer Reduktion der Aerosolverbreitung propagiert wurde, war die Verwendung von speziellen Schutzvorkehrungen während $\mathrm{HNO}$-chirurgischer Eingriffe. Absauginstrumente bei rhinochirurgischen Eingriffen [10] oder Keimbarrieren bei otochirurgischen Interventionen [7] seien hier exemplarisch genannt. $\mathrm{Ob}$, wie schnell und in welchem Umfang Op.Instrumente ersetzt wurden oder sich das operative Set-up den neuen Hygieneempfehlungen angepasst hat, ist bisher nicht flächendeckend evaluiert worden.

Um diese Entwicklung nachvollziehen zu können, sollte man sich an die Situation im März und April 2020 zurückerinnern. Die Forderung nach einem adäquaten persönlichen Schutz war durchaus gerechtfertigt, zumal sich etwa ein Drittel der HNO-ÄrztInnen einer Web-basierten Umfrage zufolge unzureichend oder gar nicht während der ersten Infektionswelle geschützt fühlten. Bei denjenigen, die sich mit SARS-CoV-2 infiziert haben, lag der Anteil bei ca. 55\% [13]. Es ist zu vermuten, dass eine Verbesserung des Schutzes von medizinischem Personal auch zu Steigerung der empfundenen Sicherheit führen wird.

\section{Maßnahmen zur Minderung des Infektionsrisikos}

Die generelle Empfehlung, Hals-NasenOhren-ärztliche Untersuchungen und Eingriffe nur nach strenger Indikationsprüfung durchzuführen, ist sicherlich während der Hochphase der ersten Infektionswelle beachtet worden [29]. Mit Rückgang der Infektionszahlen und zunehmendem Druck, wieder zur Normalität zurückzukehren, ist zu vermuten, dass die Indikationsstellung inzwischen wieder weniger restriktiv erfolgt oder in Zukunft erfolgen wird. Der Eigenschutz des medizinischen Personals wird dennoch stärker in den Fokus treten. Dies wird auch die verfügbare HNO-Ausrüstung betreffen. Die Verwendung von Videoendoskopen, bei denen der Untersucher nicht direkt vor dem Patienten positioniert sein muss, könnte die Infektionsgefahr für den Untersuchenden im Vergleich zur nicht über Video übertragenen Lupenlaryngoskopie verringern. Vergleichende Untersuchungen hierzu liegen aktuell noch nicht vor und sollten erfolgen, bevor pauschal zu einer kostenintensiven Umrüstung der Endoskope geraten werden kann. Werden wir keine Verbesserung des apparativen Schutzes erreichen, werden sich unter Umständen Untersuchende zu einer Risiko-NutzenAbwägung gezwungen sehen, die nicht hauptsächlich aus Sicht der Patienten stattfindet, sondern zunehmend eine Risikokalkulation aus Sicht der Behandler wird. Dies könnte dazu führen, dass risikoreiche Untersuchungen und Eingriffe nicht erfolgen und die gewohnte Qualität der Diagnostik und Therapie sinkt (z.B. kompletter HNO-Status vs. alleinige symptombezogene Otoskopie).

Mit Anstieg der PCR-Testkapazitäten für SARS-CoV-2 und Verfügbarkeit von Antigentests ist eine weitere Sicherheitssäule für das Personal im Gesundheitssektor hinzugekommen. Bereits frühzeitig im Mai 2020 wurde durch eine kanadische Autorengruppe [23] der Gedanke (lediglich in einem Nebensatz) geäußert, dass die Testung auf SARS-CoV-2 (Patienten und Behandler, Anm. der Autoren) zum Schutz der Beschäftigten im Gesundheitssystem beitragen könnte. Zum damaligen Zeitpunkt war die Kapazität 
HNO 2021 · 69:615-622 https://doi.org/10.1007/s00106-021-01015-2

(c) Springer Medizin Verlag GmbH, ein Teil von Springer Nature 2021

\section{Herzog · A. G. Beule · J.-C. Lüers · O. Guntinas-Lichius · D. Grafmans · T. Deitmer}

\section{Das erste Jahr der Pandemie mit SARS-CoV-2 - Auswirkungen auf die Hals-Nasen-Ohren-Heilkunde}

\section{Zusammenfassung}

Hintergrund. Das erste Jahr der Pandemie mit dem Erreger SARS-CoV-2 (,severe acute respiratory syndrome coronavirus $2^{\prime \prime}$ ) hat unser Gesundheitssystem bereits stark verändert und wird dies auch weiterhin tun. Durch die erhöhte Virusexposition in der Schleimhaut der oberen Luftwege scheinen HNO-ÄrztInnen besonders gefährdet zu sein, sich zu infizieren. Die vorliegende Übersichtsarbeit soll die Auswirkungen auf die HNO-Heilkunde darstellen und eine Einschätzung des Infektionsrisikos für HNOÄrztInnen geben.

Methoden. Es erfolgte eine Recherche in den Datenbanken PubMed, medRxiv und bioRxiv sowie im Deutschen Ärzteblatt und auf den Websites des Robert Koch-Instituts und der Johns Hopkins University nach den einschlägigen deutschen und englischen
Fachtermini im Bereich HNO, SARS-CoV-2, Risiko und Infektion.

Ergebnisse. Schutzempfehlungen für HNOÄrzte beinhalten neben den allgemeinen Hygieneregeln das konsequente Tragen von höherwertigen Masken (FFP2/3) in der Routinetätigkeit. Bei Kontakt zu Patienten mit COVID-19 (",coronavirus disease 2019“) wird empfohlen, diese Schutzausrüstung um Augenschutz, Kittel, Handschuhe und Haube zu erweitern. Es liegen Empfehlungen internationaler HNO-Fachgesellschaften für bestimmte HNO-ärztliche Tätigkeiten vor (z. B. Tracheotomie, NNH-Chirurgie), wobei neben dem Schutz des Op.-Teams die Reduktion der Aerosolausbreitung essenziell ist. Eine Testung des medizinischen Personals und der Patienten auf SARS-CoV-2 kann helfen, das Infektionsrisiko zu verringern. Durch eine
Impfung würde eine zusätzliche Sicherheit für HNO-Ärzte, die einer erhöhten Aerosolexposition ausgesetzt sind, entstehen. Nach vorliegenden Daten scheinen HNO-ÄrztInnen hinsichtlich eines Infektionsrisikos zu den besonders stark gefährdeten Berufsgruppen zu gehören.

\section{Schlussfolgerung. Der konsequente}

Eigenschutz, häufige Testungen von Patienten und Therapeuten und die in Aussicht gestellte Impfung gegen SARS-CoV-2 können einen adäquaten Schutz für besonders exponierte Berufsgruppen bieten.

Schlüsselwörter

Otorhinolaryngologie · COVID-19 · Corona .

Aerosol · Schutz

\section{The first year of the SARS-CoV-2 pandemic-impact on otorhinolaryngology}

\section{Abstract}

Background. The first year of the severe acute respiratory syndrome coronavirus 2 (SARSCoV-2) pandemic has already affected our public health care system to an enormous extent and will continue to do so in the future. Otorhinolaryngologists (ORLs) are suspected to be at high risk of infection, due to the high viral load in the mucosa of the upper airways. The current review evaluates the impact of the pandemic on ORLs' activities and assesses the risk infection.

Methods. A selective literature research was conducted using relevant English and German terms for ORL, SARS-CoV-2, risk, and infection at PubMed, medRxiv, and bioRxiv, as well as in the Deutsches Ärzteblatt and on the websites of the Robert Koch Institute and the Johns Hopkins University.

Results. Protection recommendations for ORL include general hygiene measures and wearing KN95 masks for routine professional activities. When in contact with coronavirus disease 2019 (COVID19) patients, it is recommended to extend the personal protective equipment by eye protection, gloves, cap, and gown. International otorhinolaryngology societies have released guidelines for procedures (e.g., tracheostomy, sinus surgery), propagating personal protection for the surgical team and reduction of aerosols. Testing for SARS-CoV-2 in patients and medical staff can contribute to reducing the risk of infection. Vaccination would provide some additional protection for ORLs and other health care professionals with increased exposure to aerosols. There is increasing evidence that ORLs are at a high risk of contracting SARS-CoV-2.

Conclusion. Consequent personal protection, frequent testing of patients and health care professionals, and the promised SARS-CoV-2 vaccinations may provide adequate protection for highly exposed persons.

Keywords

ENT · COVID-19 · Corona · Aerosol · Protection für umfangreiche PCR-Untersuchungen noch gar nicht vorhanden, was aus heutiger Sicht nur noch schwer nachzuvollziehen ist.

Die im Vergleich zur ersten Welle eingeschränkten materiellen Ressourcen sind in der zweiten Infektionswelle bisher in Deutschland nicht zu erkennen. Ebenso sind national die Testkapazitäten um ein Vielfaches erhöht worden, was es uns ermöglicht, Patienten vor einem geplanten stationären Aufenthalt routinemäßig mittels PCR zu testen oder per Antigentest vor einem ambulanten Kontakt bei Bedarf zu screenen. Unklar dagegen ist die Gegenfinanzierung dieses erheblichen Mehraufwands, der alle Sparten des Gesundheitssystems erheblich belasten wird.

Aktuell besteht eine multifaktorielle Schutzstrategie in Deutschland gegen SARS-CoV-2 [17].

1. Allgemeine Hygienemaßnahmen

(Abstand, Händedesinfektion, MundNase-Schutz oder FFP2/3-Masken, wie in den meisten Kliniken inzwischen üblich)

2. Patientenscreening

3. Personalscreening

4. Der Pandemie angepasste Indikationsstellungen

5. Reduktion von Kontaktpersonen

6. Umsetzung von klinikindividuellen Hygiene- und Vorsorgekonzepten (baulich, technisch, logistisch, z. B. bauliche Separierung von COVIDStationen, getrennte Lüftungssyste- 

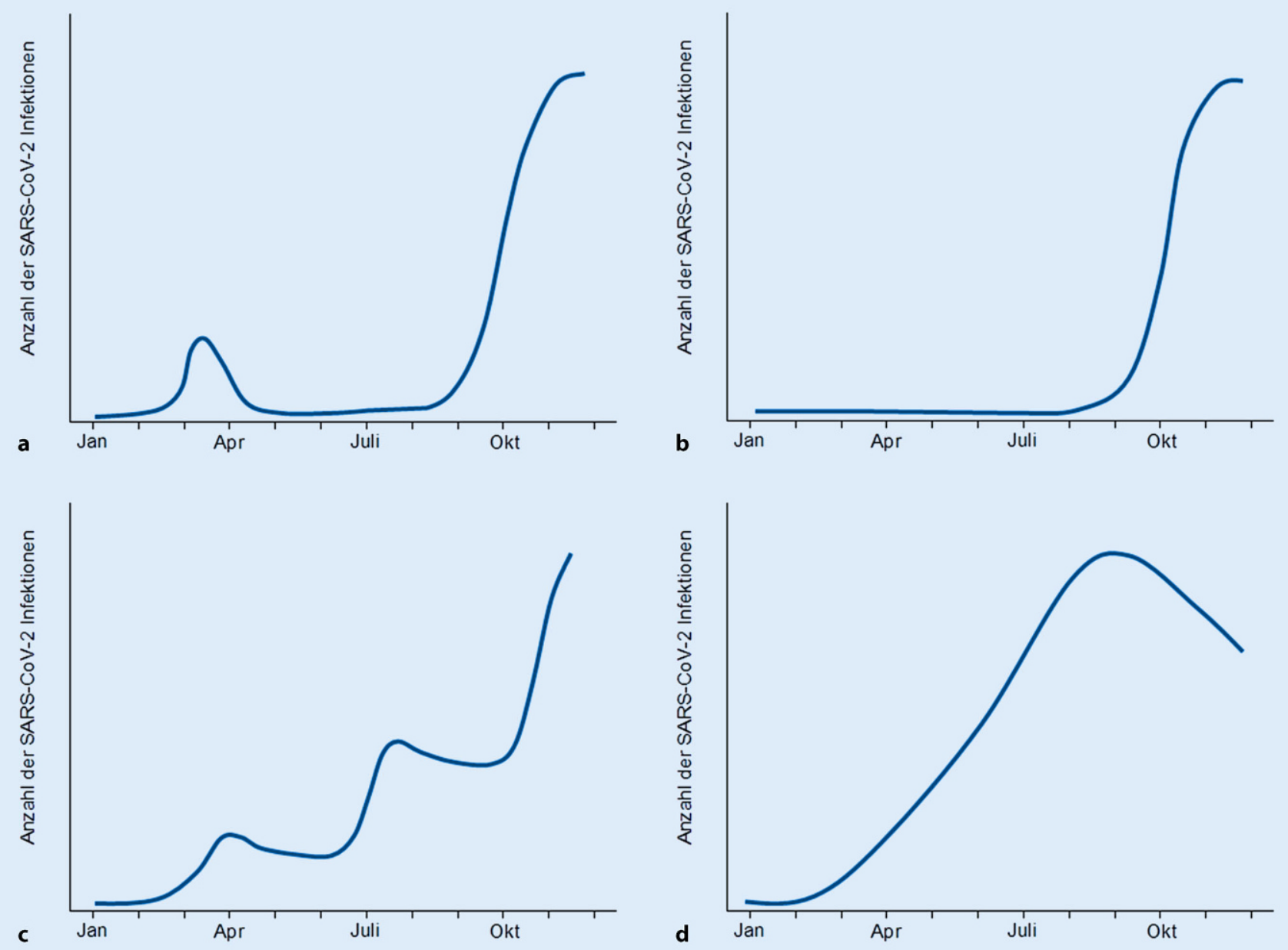

Abb. 1 \ Schematische Verläufe der Infektionen mit SARS-CoV-2 im Jahr 2020. a Erste Welle im Frühjahr mit starkem Rückgang der Infektionszahlen im Sommer und starkem Anstieg in der zweiten Welle im Herbst (Länder des westlichen, mittleren und nördlichen Europas; z. B. Deutschland, Frankreich, Italien, Spanien, Großbritannien, Norwegen, Schweden, Finnland). b Kaum vorhandene erste Welle im Frühjahr mit stabiler Phase im Sommer und starker zweiter Welle im Herbst (Länder des östlichen Europas; z. B. Polen, Tschechien, Ungarn, Rumänien, Ukraine). c Erste Welle im Frühjahr mit leichtem Rückgang, aber Stagnation auf erhöhtem Niveau im Sommer und treppenförmiger Verlauf der zweiten und evtl. dritten Welle (z. B. USA:3 Wellen, Russland, Mexiko, Kanada, Peru: 2 Wellen). d Kontinuierlicher Anstieg seit Frühjahr bis Herbst und Absinken im Spätherbst (z. B. Indien, Brasilien, Venezuela, Argentinien). Die y-Achse stellt die Anzahl der Infektionen dar und ist nicht numerisch skaliert. Die Quantität des Infektionsgeschehens variiert von Land zu Land, ebenso die zeitliche Abfolge der Wellen innerhalb einzelner Länder oder Regionen, sodass die angegeben Monate als grobe Orientierung zu verstehen sind. Die Grafik soll schematisch Verlaufscluster darstellen. Quelle der nationalen Infektionsverläufe: COVID-19 Map - Johns Hopkins Coronavirus Resource Center; https://coronavirus.jhu.edu/

me, eigener Zugang, separates Pflegeund Ärztepersonal, usw.)

7. Adäquater persönlicher Schutz

8. Optimierte technische Schutzmaßnahmen in speziellen Situationen (z. B. OP)

9. Impfung gegen SARS-CoV-2

Welchen Stellenwert eine Impfung gegen SARS-CoV-2 kurz-, mittel- und langfristig haben wird, bleibt abzuwarten. Es bleibt auch ungewiss, wie sich weitere Mutationen des Virus auf das Infektions- geschehen auswirken. Deswegen werden die genannten multifaktoriellen Schutzmaßnahmen weiter von Bedeutung bleiben und in unseren Arbeitsalltag für längere Zeit (wenn nicht dauerhaft) integriert werden müssen.

\section{Sind HNO-ÄrztInnen besonders gefährdet, sich mit SARS-CoV-2 zu infizieren?}

Beim Studium der Literatur über den Zusammenhang zwischen berufsbedingter
Aerosolexposition und der Möglichkeit, sich mit SARS-CoV-2 zu infizieren, fällt auf, dass zahlreiche Arbeiten zur Aerosolentstehung und daraus resultierenden Schutzmaßnahmen existieren. Es liegen nur wenige Daten über die Infektionshäufigkeit von MitarbeiterInnen des Gesundheitssystems und Hals-Nasen-Ohren-ÄrztInnen im Speziellen vor.

Eine der ersten Arbeiten wurde von Sowerby et al. im Frühjahr 2020 mit retrospektiven Daten der ersten Infektionswelle (Januar - April 2020) publiziert 
[41]. Berichtet wurde hier von 361 infizierten und 24 an COVID-19 verstorbenen Hals-Nasen-Ohren-ÄrztInnen aus 19 Ländern. Allerdings liegt die methodische Schwäche dieser Studie darin, dass es sich um individuelle Zählungen aus den einzelnen Ländern handelt, die teilweise nicht durch eine Befragung oder Datenabfrage eines Registers zustande gekommen sind, sondern auf der Recherche einzelner Personen oder Gruppen beruhen. Des Weiteren gingen auch klinisch als COVID-19-positive Fälle eingestufte Personen ohne PCRVirus-RNA-Nachweis in die Zählung ein. Nur in 9 Ländern wurden alle positiv gemeldeten Ärzte auch per PCR oder Antikörpertest verifiziert. Mit Ausnahme einer Nation wurden keine Befragungen/Registerhebungen aller Hals-NasenOhren-ÄrztInnen eines Landes durchgeführt, weshalb keine Inzidenzwerte und Risikoeinschätzungen für Hals-Nasen-Ohren-ÄrztInnen ermittelt werden konnten.

Ein weiteres Problem bei der Interpretation der Daten liegt in der Heterogenität der Infektionsverläufe in den einzelnen Ländern. Je nach Ausmaß und zeitlichem Verlaufder Pandemie in den unterschiedlichen Ländern werden abweichende Daten zum Infektionsgeschehen zu erwarten sein. Im ersten Quartal 2020 berichteten Autoren aus China und weiteren asiatischen Ländern bereits in ersten größeren Kollektiven zu Infektionsraten bei medizinischem Personal, während Europa mitten in der ersten Welle steckte und der amerikanische Kontinent noch nicht in vollem Ausmaß betroffen war.

Ähnliches gilt sicherlich auch für die Charakteristik der auftretenden Wellen des Pandemiegeschehens.

Es lassen sich unterschiedliche Cluster von Infektionsverläufen definieren (• Abb. 1).

In Abhängigkeit von den Infektionsverläufen werden unterschiedliche Daten zu Infektionsraten bei medizinischem Personal zu ermitteln sein. Dies gilt insbesondere dann, wenn gesundheitspolitische Gegebenheiten der Länder stark voneinander abweichen.

- Konnte ein Land sein Gesundheits-

system nach der ersten Welle wieder stabilisieren und sich auf eine an- stehende zweite Welle vorbereiten (• Abb. 1a)?

- Konnten Nationen, die keine erste Welle erlebt haben, aus den Erfahrungen anderer Länder lernen und das eigene Gesundheitssystem auf die Pandemie vorbereiten (• Abb. 1b)?

- Konnten Länder ihr Gesundheitssystem trotz permanent hoher Infektionszahlen während der einzelnen Wellen stabilisieren und die Dauerbelastung des medizinischen Personals in Grenzen halten (- Abb. 1c)?

- Konnten Länder mit einem kontinuierlichen Anstieg der Infektionszahlen Personal und Logistik den steigenden Infektionszahlen anpassen (- Abb. 1d)?

- Wie werden die Gesundheitssysteme mit den gestiegenen Anforderungen dauerhaft zurechtkommen, wenn nach einer Welle kein Rückgang der Infektionszahlen auf einen sehr niedrigen Basiswert erfolgt (• Abb. 1a), sondern als Plateau weiterhin hoch bleibt und in die nächste Welle übergeht (• Abb. 1c)?

Diese Faktoren hängen für jedes Land von den individuellen Gegebenheiten des Gesundheitssystems und den gesundheitspolitischen Maßnahmen und Gegebenheiten (Lock-down, Bereitstellung von Schutzausrüstungen, Infektionskettennachverfolgung, usw.) ab, was die Interpretation von Publikationen zum Infektionsgeschehen einzelner Länder schwierig macht.

Es ist deshalb von großer Bedeutung, nationale oder regionale Daten zum Infektionsgeschehen bei medizinischem Personal zu erhalten, um das Infektionsrisiko für Hals-Nasen-Ohren-Ärzte abzuschätzen. Eine Web-basierte Erhebung aus Großbritannien [42], publiziert im August 2020, nennt 73 Teilnehmer, von denen 35 eine durch Testung bestätigte Infektion mit SARS-CoV-2 angaben (47,9\%!), wobei sicherlich von einem relativ hohen „reporting bias“ auszugehen ist. Interessant ist, dass $60,3 \%$ der infizierten britischen HNO-ÄrztInnen die Ursache ihrer eigenen Infektion im beruflichen Umfeld vermuteten, wobei keine Aussagen dazu gemacht wurden, ob infizierte Patienten, ärztliche Kollegen oder infiziertes medizinisches Personal darunter zu verstehen sind.

Eine Web-basierte Umfrage bei deutschen HNO-ÄrztInnen [13] konnte Daten von 970 Teilnehmern sammeln (ca. 1/7 aller in Deutschland gemeldeten HNO-ÄrztInnen), was im internationalen Vergleich bemerkenswert erscheint. Die Stärke dieser Befragung lag u. a. in der Tatsache, dass die nationale Fachgesellschaft (DGHNO-KHC) und der nationale Berufsverband (HNO-Berufsverband) ihre Mitglieder mehrfach erinnerten und zur Teilnahme aufriefen. So konnte bis Ende Mai 2020 ein guter Überblick über das Infektionsgeschehen der ersten 4 Monate in Deutschland ermittelt werden. 54 Hals-Nasen-OhrenÄrztInnen meldeten sich als bestätigt positiv auf SARS-CoV-2. Aufgrund der soliden Datenlage konnte ein relatives Risiko für Hals-Nasen-Ohren-ÄrztInnen im Vergleich zur deutschen Gesamtbevölkerung von 3,67 berechnet werden, sich mit SARS-CoV-2 zu infizieren. Bei $3 / 5(n=31)$ der Infizierten war die Infektionskette nicht geklärt. Von den verbleibenden 2/5 $(n=23)$ infizierten sich 5 Personen durch private Kontakte und 18 durch berufliche Tätigkeiten. Hierbei gehen 5 Infektionen auf den Kontakt zu infizierten Patienten zurück und 13 auf den Kontakt zu infiziertem Personal. Die hauptsächliche Ansteckung von HalsNasen-Ohren-ÄrztInnen durch den Umgang mit infizierten Patienten lässt sich aus diesen Daten nicht belegen (5 von $23=22 \%$ ). Allerdings fühlten sich aus der Gesamtheit der Befragten 33\% nur unzureichend oder gar nicht bei ihrer Berufsausübung geschützt. Bei den mit SARS-CoV-2 infizierten Befragten waren es $56 \%$. Es bleibt zu diskutieren, inwieweit der tatsächliche oder gefühlte Mangel an persönlicher Schutzausrüstung zu Beginn der Pandemie diese Ergebnisse beeinflusste.

Alle drei der genannten Erhebungen versuchten, über eine direkte Befragung von Hals-Nasen-Ohren-Ärzten an Infektions-, Erkrankungs- oder Sterbedaten zu gelangen. Dieser methodische Ansatz ist äußert schwierig, da in so gut wie allen Ländern detaillierte Auflistungen von Subgruppen innerhalb des medizinischen Personals nicht existie- 
ren. Der Versuch, über eine GoogleRecherche (aus Ermangelung aussagekräftiger Studien in Medline) Daten über Todesfälle aufgrund von COVID19 bei Ärzten aller Fachrichtungen zu erhalten, ist sicherlich aufsehenerregend, die Interpretation sollte jedoch mit Blick auf die Methodik der Arbeit mit Bedacht erfolgen [15]. Interessant ist, dass hiernach Allgemeinärzte die Hauptgruppe an Todesfällen darstellen (42\%), wohingegen Hals-NasenOhren-Ärzte lediglich $4 \%$ der Todesfälle ausmachen. Persönliche Nachfragen bei einem Träger der gesetzlichen Unfallversicherung, dem Erkrankungsfälle von COVID im beruflichen Kontext als Berufskrankheit oder Arbeitsunfall zu melden sind, ergaben, dass eine Identifikation von ärztlichen Fachgruppen zumindest momentan nicht möglich ist.

Es bleibt der Dynamik der Pandemie geschuldet, dass zur aktuell existenten zweiten Infektionswelle noch keine Daten zum Infektionsgeschehen im Hinblick auf betroffene Hals-Nasen-OhrenÄrztInnen vorliegen. Es ist $\mathrm{zu}$ vermuten, dass sich auch vermehrt Hals-Nasen-Ohren-ÄrztInnen mit SARS-CoV-2 infizierten, wobei von besonderem Interesse sein wird, ob die Infektionen durch den beruflichen Kontakt mit bekannten COVID-19-Patienten oder asymptomatischen Patienten stattfanden oder hauptsächlich durch Kontakte zu asymptomatischem medizinischem Personal oder im Privatbereich zu suchen sind. Ein geringer Anteil von Infektionen durch Patientenkontakt würde die Wirksamkeit der bisherigen beruflichen Präventionsmaßnahmen unterstreichen.

\section{Leben und arbeiten mit einem erhöhten Infektions- risiko für Beschäftigte im Gesundheitssystem}

Publikationen zeigen, dass Beschäftigte im Gesundheitssystem in vielen Ländern ein erhöhtes Risiko aufweisen, sich mit SARS-CoV-2 zu infizieren und an COVID-19 zu erkranken [3, 31, 34, 39, 46]. Viele Studien sehen ein erhöhtes Risiko für Klinikpersonal, das in unmittelbarem Kontakt zu COVID-19-Patienten steht. Allerdings weisen die Daten darauf hin, dass auch im ambulanten Bereich das Risiko erhöht ist, sich mit SARS-CoV-2 zu infizieren, wofür auch die Ergebnisse der deutschen Umfrage bei Hals-Nasen-Ohren-ÄrztInnen sprechen (57\% der infizierten Hals-Nasen-Ohren-ÄrztInnen in einer Praxis tätig) [13].

Trotz der weiter existierenden Pandemie werden wir wieder zur Normalität im Gesundheitssystem zurückkehren müssen, um unseren Hals-Nasen-Ohren-Patienten, deren Behandlung aufgrund der Pandemie aufgeschoben wurde, wieder die gewohnte medizinische Sorgfalt bieten zu können. Die genannten Schutzstrategien werden weiterhin von Bedeutung sein [17].

Zusätzlich hat sich inzwischen die Chance eröffnet, gegen SARS-CoV-2 zu impfen. Da Beschäftigte im medizinischen Sektor ein erhöhtes Infektionsrisiko haben und ihre Arbeitskraft während der Pandemie weiter stark benötigt wird, wird propagiert, diese Personen priorisiert zu impfen. Nach den aktuellen Empfehlungen der Ständigen Impfkommission vom 14.01.2021 wird die Tätigkeit in einer Hals-Nasen-Ohren-ärztlichen Praxis mit der Risikostufe 2 klassifiziert (hohes Expositionsrisiko). Tätigkeiten mit besonders starker Aerosolgeneration (angeführt: Laryngoskopie, Interpretation: alle operativen Eingriffe an der respiratorischen Schleimhaut) werden zur Risikostufe 1 gezählt (besonders hohes Expositionsrisiko) [40]. Somit wären entsprechende Tätigkeiten als Argument anzuführen, Hals-Nasen-Ohren-Ärzte priorisiert zu impfen, wie es seitens der DGHNO-KHC und des HNO-Berufsverbands vorgeschlagen wurde.

Als Gegenargument kann angesehen werden, dass das Infektionsgeschehen während der zweiten Infektionswelle nicht vornehmlich auf Gesundheitseinrichtungen konzentriert war, sondern gehäuft private Kontakte betroffen waren und sind [21]. Dies scheint der Strategie der Priorisierung von Gesundheitspersonal $\mathrm{zu}$ widersprechen. Zum Infektionsstatus der deutschen HNOÄrztInnen während der zweiten Infektionswelle kann noch keine Aussage gemacht werden, da eine Umfrage im Auftrag der DGHNO-KHC und des HNO-Berufsverbands nach infizierten
HNO-ÄrztInnen noch geschaltet und noch nicht abschließend ausgewertet ist. Bedacht werden sollte, dass sich auch MitarbeiterInnen des Gesundheitssystems durch private Kontakte infizieren können. Im Vergleich zu Infizierten, welche nicht im Gesundheitssystem arbeiten, besteht hier die Gefahr, dass der Erreger durch berufliche Kontakte auf gesundheitliche Risikogruppen übertragen werden kann, was verheerende Folgen haben kann. Aber auch wenn diese Infizierten aus dem Gesundheitsbereich das Virus durch Einhaltung von Isolation (wenn bekannt infiziert) oder durch konsequente Hygienemaßnahmen (wenn unbekannt infiziert und deshalb weiter arbeitend) nicht zwangsläufig auf Patienten und MitarbeiterInnen weiterverbreiten werden, so fallen PflegerInnen und ÄrztInnen krankheitsbedingt aus, wenn sie symptomatisch werden und stehen nicht mehr für die Krankenversorgung zur Verfügung. Dies würde die generell schon knappen Personalressourcen im Gesundheitssystem weiter verschärfen. In diesem argumentativen Kontext ist eine priorisierte Impfung bei medizinischem Personal sinnvoll.

\section{Abschließende Gedanken und Ausblick}

Die Betrachtung des bisherigen Pandemieverlaufs zeigt die rasante Entwicklung des Infektionsgeschehens, aber auch der medizinischen und gesundheitspolitischen Veränderungen. Eine Erkrankung, die vor einem Jahr noch gar nicht existierte, führt jetzt dazu, dass wir unser komplettes medizinisches Handeln überdenken und anpassen müssen. Der Wandel wird auch die Hals-Nasen-Ohren-Heilkunde betreffen, und jeder einzelne Akteur ist in der Pflicht, sowohl sein Privatleben als auch seinen Berufsalltag auf die Gegebenheiten abzustimmen. Schutzkonzepte können von Fachgesellschaften und Spezialisten entworfen und deren Umsetzung behördlich vorgeschrieben werden - für eine effektive Bekämpfung der Pandemie bedarf es der konsequenten Beteiligung jedes Einzelnen. Es wird sicher noch Zeit brauchen, bis Hygienekonzepte nicht nur mehr oder weniger bereitwillig und um- 
sichtig bei der Arbeit befolgt werden, sondern auch zum Bestandteil unseres beruflichen und privaten Lebens geworden sind.

In diesem Zusammenhang erscheint es angebracht, über mögliche Strukturen im deutschen Gesundheitssystem nachzudenken, die es uns ermöglichen, auch bei zukünftigen auftretenden Infektionswellen eine geregelte Patientenversorgung parallel zur Versorgung von COVID-19-Patienten zu gewährleisten. In der aktuellen zweiten Infektionswelle wurde/wird in vielen Kliniken Personal, welches aufgrund der ausgesetzten Routineversorgung freigesetzt wurde, auf COVID-Stationen eingesetzt. Diese Maßnahmen sind den knappen Personalressourcen und dem übermäßigen Patientenaufkommen geschuldet. Man muss sich kritisch fragen, ob bei einer möglichen dritten Infektionswelle erneut ein Herunterfahren elektiver Behandlungen erfolgen kann. Es könnte für größere Kliniken sinnvoller erscheinen, eine komplett autarke Infektionseinheit vorzuhalten, welche personell und logistisch unabhängig vom restlichen Klinikbetrieb COVID-19-Patienten behandeln kann. Der Hauptklinik wird noch ausreichend Personal zur Verfügung stehen, um ohne Einschränkungen der regulären Krankenversorgung nachzukommen. Sollte das Personal der Infektionseinheit nicht gebraucht werden, würde es in die regulären Abteilungen einer Klinik zurückgeführt werden. Essenziell wäre hierbei, dass ausreichend Personal zur Verfügung steht, um innerhalb weniger Stunden aus dem Regelbetrieb in die Infektionseinheit zu wechseln, ohne den Betrieb des restlichen Klinikums zu gefährden. Eine personelle Aufstockung wäre grundlegend für einen solchen Ansatz. Um solche Konzepte jedoch umzusetzen, bedarf es einer Akzeptanz der Verantwortlichen, das bisherige Versorgungssystem kritisch zu hinterfragen und entsprechende Konsequenzen $\mathrm{zu}$ ziehen.

\section{Korrespondenzadresse}

\section{PD Dr. M. Herzog}

Klinik für Hals-Nasen-Ohrenheilkunde, Kopfund Halschirurgie, Carl-Thiem-Klinikum Thiemstraße 111, 03048 Cottbus, Deutschland M.Herzog@ctk.de

\section{Einhaltung ethischer Richtlinien}

Interessenkonflikt. M. Herzog, A. G. Beule, J.-C. Lüers, O. Guntinas-Lichius, D. Grafmans und T. Deitmer geben an, dass kein Interessenkonflikt besteht.

Für diesen Beitrag wurden von den Autoren keine Studien an Menschen oder Tieren durchgeführt. Für die aufgeführten Studien gelten die jeweils dort angegebenen ethischen Richtlinien.

\section{Literatur}

1. Böhmer MM, Buchholz U, Corman VM et al (2020) Investigation of a COVID-19 outbreak in Germany resulting from a single travel-associated primary case. A case series. Lancet Infect Dis 20(8):920-928. https://doi.org/10.1016/S1473-3099(20)30314-5

2. Bundesagentur für Arbeitsschutz und Arbeitsmedizin (2020) Empfehlungen der BAuA und des ad-Hoc AK "Covid-19" des ABAS zum Einsatz von Schutzmasken im Zusammenhang mit SARS-CoV-2. https://www.baua.de/DE/Themen/ Arbeitsgestaltung-im-Betrieb/Coronavirus/pdf/ Schutzmasken.pdf?_blob=publicationFile\& $\mathrm{v}=$ 17. Stand:22.1.2021.Zugegriffen:22.2.2021

3. Canova V, Lederer Schläpfer H, Piso RJ et al (2020) Transmission risk of SARS-CoV-2 to healthcare workers-observational results of a primary care hospital contact tracing. Swiss Med Wkly 150:w20257. https://doi.org/10.4414/smw.2020. 20257

4. Chan JYK, Wong EWY, Lam W (2020) Practical aspects of otolaryngologic clinical services during the 2019 novel Coronavirus epidemic. An experience in Hong kong. JAMA Otolaryngol Head NeckSurg. https://doi.org/10.1001/jamaoto.2020. 0488

5. Chen JX, Workman AD, Chari DA et al (2020) Improving barrier drapes for the mitigation of aerosol and particulate spread during mastoidectomy. Otol Neurotol. https://doi.org/10.1097/ MAO.0000000000002906

6. China Newsweek View inews.qq.com/a/2020012 5A07TT200?uid=\&devid=BDFE70CD-5BF1-470291B7-329F20A6E839\&qimei=bdfe70cd-5bf1-470 2-91b7-329f20a6e839.Zugegriffen:25.01.2020

7. Cottrell J, Lui J, Le T, Chen J (2020) An operative barrier system for skull base and mastoid surgery. Creating a safe operative theatre in the era of COVID-19. J Otolaryngol 49(1):71. https://doi.org/ 10.1186/s40463-020-00471-0

8. Crossley J, Clark C, Brody F, Maxwell JH (2020) Surgical considerations for an awake tracheotomy during the COVID-19 pandemic. J Laparoendosc Adv Surg Tech A. https://doi.org/10.1089/lap. 2020.0239

9. David AP, Russell MD, El-Sayed IH, Russell MS (2020) Tracheostomy guidelines developed at a large academic medical center during the COVID-19 pandemic. Head Neck. https://doi.org/10.1002/ hed.26191

10. Dharmarajan H, Freiser ME, Sim E et al (2020) Droplet and aerosol generation with endonasal surgery. Methods to mitigate risk during the COVID-19 pandemic. Otolaryngol Head Neck Surg. https://doi.org/10.1177/0194599820949802

11. Frazier KM, Hooper JE, Mostafa HH, Stewart CM (2020) SARS-CoV-2 virus isolated from the mastoid and middle ear. Implications for COVID-19 precautions during ear surgery. JAMA Otolaryngol Head Neck Surg. https://doi.org/10.1001/jamaoto. 2020.1922

12. Goldman RA, Swendseid B, Chan JYK et al (2020) Tracheostomy management during the COVID-19 pandemic. Otolaryngol Head Neck Surg. https:// doi.org/10.1177/0194599820923632

13. Herzog M, Beule AG, Lüers J-C, Guntinas-Lichius $O$, Sowerby LJ, Grafmans D (2020) Results of a national web-based survey on the SARS-CoV-2 infectious state of otorhinolaryngologists in Germany. Eur Arch Otorhinolaryngol. https://doi.org/10.1007/ s00405-020-06345-5

14. Howard BE (2020) High-risk aerosol-generating procedures in COVID-19. Respiratory protective equipment considerations. Otolaryngol Head Neck Surg 163(1):98-103. https://doi.org/10. 1177/0194599820927335

15. Ing EB, Xu QA, Salimi A, Torun N (2020) Physician deaths from corona virus (COVID-19) disease. Occup Med 70(5):370-374. https://doi.org/10. 1093/occmed/kqaa088

16. Judson SD, van Doremalen N, Munster VJ (2020) Stability and viability of SARS-CoV-2. Reply. N Engl JMed. https://doi.org/10.1056/NEJMc2007942

17. Jung F, Krieger V, HufertFT, Küpper J-H (2020) How we should respond to the Coronavirus SARS-CoV-2 outbreak. A German perspective. Clin Hemorheol Microcirc 74(4):363-372. https://doi.org/10.3233/ $\mathrm{CH}-209004$

18. Kay JK, Parsel SM, Marsh JJ, McWhorter AJ, Friedlander PL (2020) Risk of SARS-CoV-2 transmission during flexible laryngoscopy. A systematic review. JAMA Otolaryngol Head Neck Surg 146(9):851-856. https://doi.org/10.1001/ jamaoto.2020.1973

19. Kligerman MP, Vukkadala N, Tsang RKY et al (2020) Managing the head and neck cancer patient with tracheostomy or laryngectomy during the COVID19 pandemic. Head Neck. https://doi.org/10.1002/ hed.26171

20. Krajewska J, Krajewski W, Zub K, Zatoński T (2020) COVID-19 in otolaryngologist practice. A review of current knowledge. Eur Arch Otorhinolaryngol. https://doi.org/10.1007/s00405-020-05968-y

21. Kramer $R$, Klingeberg $A$, Diercke $M$, Claus $H$ et al (2020) COVID-19-Analysis of incident cases reported within the German healthcare system. Dtsch Arztebl Int 117:809-810

22. Kuhar HN, Heilingoetter A, Bergman M, Worobetz N, Chiang T, Matrka L (2020) Otolaryngology in the time of corona. Assessing operative impact and risk during the COVID-19 crisis. Otolaryngol Head Neck Surg 163(2):307-315. https://doi.org/ 10.1177/0194599820930214

23. Lammers MJW, Lea J, Westerberg BD (2020) Guidance for otolaryngology health care workers performing aerosol generating medical procedures during the COVID-19 pandemic. J Otolaryngol Head Neck Surg 49(1):36. https://doi.org/10.1186/ s40463-020-00429-2

24. LeConte B, Low GMI, Citardi MJ, Yao WC, Eguia AA, Luong AU (2020) Aerosol generation with common rhinologic devices. Cadaveric study conducted 
in a surgical suite. Int Forum Allergy Rhinol 10(11):1261-1263. https://doi.org/10.1002/alr. 22679

25. Licina A, Silvers A (2020) Use of powered airpurifying respirator(PAPR) as part of protective equipment against SARS-CoV-2-a narrative review and critical appraisal of evidence. Am J Infect Control. https://doi.org/10.1016/j.ajic.2020.11. 009

26. Licina A, Silvers A, Stuart RL (2020) Use of powered air-purifying respirator (PAPR) by healthcare workers for preventing highly infectious viral diseases-a systematic review of evidence. Syst Rev 9(1):173. https://doi.org/10.1186/s13643-02001431-5

27. Lindemann J, Böhm F, Hoffmann T et al (2020) Chirurgische Aspekte zur Tracheostomie bei Covid 19 positiven Patienten. Laryngorhinootologie 99:282-284

28. Loth AG, Guderian DB, Haake B, Zacharowski $K$ Stöver T, Leinung M (2020) Aerosol exposure during surgical tracheotomy in SARS-CoV-2 positive patients. Shock. https://doi.org/10.1097/ SHK.0000000000001655

29. Lüers J-C, Klußmann JP, Guntinas-Lichius O (2020) Die Covid-19-Pandemie und das HNO-Fachgebiet. Worauf kommt es aktuell an? Laryngorhinootologie. https://doi.org/10.1055/a-1095-2344

30. Mick P, Murphy R (2020) Aerosol-generating otolaryngology procedures and the need for enhanced PPE during the COVID-19 pandemic. A literature review. J Otolaryngol Head Neck Surg 49(1):29. https://doi.org/10.1186/s40463-02000424-7

31. Möhner M, Wolik A (2020) Differences in COVID19 risk between occupational groups and employment sectors in Germany. Dtsch Arztebl Int 117:641-642

32. Morawska L, Tang JW, Bahnfleth Wet al (2020) How can airborne transmission of COVID-19 indoors be minimised? Environ Int 142:105832. https://doi. org/10.1016/j.envint.2020.105832

33. Murr A, Lenze NR, Brown WC et al (2020) Quantification of aerosol particle concentrations during endoscopic sinonasal surgery in the operating room. Am J Rhinol Allergy. https://doi. org/10.1177/1945892420962335

34. Nienhaus A, Hod R (2020) COVID-19 among health workers in Germany and Malaysia. Int J Environ Res Public Health 17(13):4881. https://doi.org/10. 3390/ijerph17134881

35. Panuganti BA, Pang J, Califano J, Chan JYK (2020) Procedural precautions and personal protective equipment during head and neck instrumentation in the COVID-19 era. Head Neck 42(7):1645-1651 https://doi.org/10.1002/hed.26220

36. Patel ZM, Fernandez-Miranda J, Hwang PH, Nayak JV et al. (2020) Letter: Precautions for endoscopic transnasal skull base surgery during the COVID-19 pandemic. Neurosurgery 87(1):E66-E67. https://doi.org/10.1093/neuros/ nyaa 125

37. Portugal LG, Adams DR, Baroody FM, Agrawal N (2020) A surgical safety checklist for performing tracheotomy in patients with Coronavirus disease 19. Otolaryngol Head Neck Surg. https://doi.org/ 10.1177/0194599820922981

38. Rameau A, Young VN, Amin MR, Sulica L (2020) Flexible laryngoscopy and COVID-19. Otolaryngol Head Neck Surg. https://doi.org/10. 1177/0194599820921395

39. Rivett L, Sridhar S, Sparkes D et al (2020) Screening of healthcare workers for SARS-CoV-2 highlights the role of asymptomatic carriage in COVID-19 transmission. eLife. https://doi.org/10.7554/eLife. 58728

40. Seedat J, Winkler M (2021) Beschluss der STIKO zur 1. Aktualisierung der COVID-19-Impfempfehlung Epidemiologische Bulletin, Bd.2

41. Sowerby LJ, Stephenson K, Dickie A et al (2020) International registry of otolaryngologist-head and neck surgeons with COVID-19. Int Forum Allergy Rhinol.https://doi.org/10.1002/alr.22677

42. Stephenson K, Sowerby LJ, Hopkins C, Kumar N (2020) The UK national registry of ENT surgeons with coronavirus disease 2019. J Laryngol Otol 134(8):665-669. https://doi.org/10.1017/ S0022215120001747

43. Syamal M (2020) Literature-guided recommendations for otolaryngologists during the COVID-19 pandemic. A contemporary review. Laryngoscope Investig Otolaryngol 5(3):432-437. https://doi. org/10.1002/lio2.389

44. Thamboo A, Lea J, Sommer DD et al. (2020) Clinical evidence based review and recommendations of aerosol generating medical procedures in otolaryngology - head and neck surgery during the COVID-19 pandemic. J Otolaryngol Head Neck Surg 49:28. https://doi.org/10.1186/s40463-02000425-6

45. Thomas-Rüddel D, Winning J, Dickmann $P$ et al (2020) "Coronavirus disease 2019" (COVID-19). Update für Anästhesisten und Intensivmediziner März 2020. Anaesthesist. https://doi.org/10.1007/ s00101-020-00760-3

46. Tosoni A, Rizzatti G, Nicolotti N et al (2020) Hospital reengineering against COVID-19 outbreak. 1-month experience of an Italian tertiary care center. Eur Rev Med Pharmacol Sci 24(15):8202-8209. https://doi.org/10.26355/eurrev_202008_22509

47. van Doremalen N, Bushmaker T, Morris DH et al (2020) Aerosol and surface stability of SARSCoV-2 as compared with SARS-CoV-1. N Engl J Med 382(16):1564-1567.https://doi.org/10.1056/ NEJMc2004973

48. Workman AD, Jafari A, Welling DB et al (2020) Airborne aerosol generation during endonasal procedures in the era of COVID-19. Risks and recommendations. Otolaryngol Head Neck Surg 163(3):465-470. https://doi.org/10.1177/ 0194599820931805

49. Workman AD, Welling DB, Carter BS et al (2020) Endonasal instrumentation and aerosolization risk in the era of COVID-19. Simulation, literature review, and proposed mitigation strategies. Int Forum Allergy Rhinol 10(7):798-805. https://doi. org/10.1002/alr.22577

50. Zou L, Ruan F, Huang Metal (2020) SARS-CoV-2 viral load in upper respiratory specimens of infected patients. NEngl J Med 382(12):1177-1179. https:// doi.org/10.1056/NEJMc2001737

\section{Wenn man die Gefahr rie- chen kann}

Forscher finden Zellen in der Nase, die giftiges Molekül wahrnehmen können

Ein Forschungsteam um den Homburger Physiologen Prof. Frank Zufall hat in der $\mathrm{Na}$ se von Mäusen Sinneszellen entdeckt, die auf eine steigende SchwefelwasserstoffKonzentration reagieren und daraufhin eine Stressreaktion auslösen.

$\mathrm{H}_{2} \mathrm{~S}$ ist eine der gefährlichsten biologisch produzierten Substanzen und kann zur Hemmung der intrazellären Atmung und damit zum Tod führen. Es entsteht dort, wo Bakterien keinen Sauerstoff verstoffwechseln können, und ist damit gerade für Tiere, die Höhlen bauen, sehr gefährlich.

Die Forscher der Saar-Universität haben anhand von Mäusen festgestellt, dass es einen Mechanismus gibt, der solche Gefahren wahrnehmen und daraufhin Abwehrmechanismen aktivieren kann. Wenn Schwefelwasserstoffmoleküle an die so genannten „Typ-B-Zellen“ in der Nase der Mäuse gelangen, wird der Ort als abstoßend empfunden und im Gehirn abgespeichert. Begleitet wird diese Überlebensstrategie von so genanntem "self-grooming behaviour" (eine Art zwanghaftes Putzen) und der Freisetzung von Stresshormonen. Die Ergebnisse dieser Grundlagenforschung sind von großer Bedeutung für das evolutionäre Verständnis des Geruchssinns und ein wichtiger Hinweis darauf, wie Pathogene von unseren Sinnesorganen detektiert werden können, um wichtige Abwehrreaktionen hervorzurufen.

\section{Originalpublikation:}

Kohei Koike et al (2021) Danger perception and stress response through an olfactory sensor for the bacterial metabolite hydrogen sulfide. Neuron.

Online first: 10.1016/j.neuron.2021.05.032

Quelle: Universität des Saarlandes (www.uni-saarland.de) 